WALTER WENZEL

walterwenzelleipzig@t-online.de

Leipzig, Deutschland
https://doi.org/10.17651/ONOMAST.65.1.5

Onomastica LXV, 2021

PL ISSN 0078-4648

\title{
DIE ALTSORBISCHEN OSĚK- UND OSĚČ- NAMEN
}

Schlüsselwörter: Namenforschung, Ortsnamen, slawisch, altsorbisch

Die im Titel genannten altsorb. OrtsN (= Ortsnamen) lassen sich aufgrund ihrer Bedeutung und Struktur als eine eigene Gruppe auffassen, die nicht nur in namenkundlicher, sondern auch in siedlungsgeschichtlicher Hinsicht besondere Beachtung verdient. Im System der altsorbischen OrtsN ordnen sie sich nach der neuesten Klassifikation in die vierte Subklasse als Typ 4.3. und Typ 4.4. ein. *Osék weist die Struktur Präfix + Nichtpersonenbezeichnung + Nullsuffix auf, *Osěč < $*$ Osěk $+j b$ die Struktur Präfix + Nichtpersonenbezeichnung + Suffix. Das anlautende *O- wird in beiden Fällen als Ortsnamenpräfix gewertet, geht man jedoch schon von einem urslaw. Substantiv *osěkb aus, wäre hier ein Nullsuffix anzusetzen. In der semantischen Subklassifikation schließen sie sich als eigene kleine Gruppe den Wald- und Rodungsnamen an (Wenzel, 2021, S. 10-20). Beide Namen beruhen auf den urslaw. Verben *sěkati, *sěkti, präfigiert *osěkati, *osěkti. Deren einzelsprachliche Kontinuanten lauten im Tschech. osekat 'abhauen, behauen', osekat větve se stromu 'einen Baum abästen, behauen, stutzen', poln. osiekać, osiec 'behauen', russ. obsěkat' 'behauen, bekappen, (be)schneiden [Äste], osěčbsja 'sich umzäunen, sich mit einem Verhau umgeben', dazu das Substantiv osěk 'Verhak, Verhau, Zaun, leichte Verschanzung, Palisadenwerk'.

Hinsichtlich ihrer Bedeutung lassen sich die im Titel genannten Bildungen Osěk und Osěč genauer als Namen bestimmen, die auf einen Grenzverhau Bezug nehmen. Das Wesen einer solchen Art der Grenzmarkierung und -sicherung beschreibt treffend das nachfolgende Zitat: „Offensichtlich war es im frühen und hohen Mittelalter in weiten Teilen Ostmitteleuropas üblich, ganze Wälder oder Waldränder an den Innenseiten der Siedlungskammern in ein bis zwei Metern Höhe zu kappen und die ineinander verkeilten Bäume zusätzlich durch Brombeeren, Heckenrosen und anderes Dornengestrüpp unwegbar zu machen. Eine solche Gestaltung von Grenzgebieten zwischen slawischen Siedlungsgebieten durch künstliche Hindernisse in der oben beschriebenen Form, als Zusatz zu Grenzwäldern und die darüberhinausgehende Verödung ganzer Grenzregionen sowie der Überwachung und Pflege durch speziell abgestellte 
Bevölkerungsgruppen scheint im Verlaufe des hohen Mittelalters üblich und durchaus effizient gewesen zu sein" (Hardt, 2019, S. 174).

Aufgabe dieses Beitrages ist es, alle OrtsN mit der Bedeutung 'Grenzverhau' im altsorb. Sprachraum zu ermitteln, historisch zu dokumentieren, ihre Lage kurz zu charakterisieren und nach Möglichkeit einer bestimmten Siedlungskammer zuzuordnen. Grundlage hierfür bilden das Kompendium von Ernst Eichler „Slawische Ortsnamen zwischen Saale und Neiße“ sowie die Beschreibung der einzelnen altsorb. Stammesgebiete durch den Verfasser in mehreren Büchern. ${ }^{1}$ Die Untersuchung beginnt im äußersten Südwesten des altsorb. Sprachgebietes mit dem Grenzverhau von Oschitz bei Schleiz in Ostthüringen und endet im Osten an der Gaugrenze von Selpoli. Darüber hinaus bleibt ein Blick auf entsprechende OrtsN in Nordostbayern sowie in Böhmen und Mähren zu werfen, da diese Großregionen im frühen Mittelalter in enger Verbindung mit dem altwestsorbischen Sprachraum zwischen Elbe und Saale standen.

Oschitz, sw. Schleiz, 1333 Oschicz, 1358 Aschicz, Ossciz, 1416 Osschicz, aso. *Osěč (Eichler, 1985-2009, Bd. III, S. 43). Der Ort liegt am äußersten Südwestrand des altsorb. Sprachraumes. Eine genauere Beschreibung der Grenzsituation hier ist nicht möglich, da keine entsprechende Monographie dieses Gebietes vorliegt.

Ossig, ssw. Zeitz, 1121 Ozzek, 1151 Ozzek, 1256 Ozzek, 1380 Osseck, 16. Jh. Ossigk (Eichler, 1985-2009, Bd. III, S. 44). Ossig liegt an einem kleinen Bach am Südrand des Gaues Puonzowa (Wenzel, 2019, S. 179, K. 3). Durch das Dorf führt heute eine Straße von Zeitz in Richtung Gera. Die lokale Altstraßenforschung hätte zu klären, ob es sich hier um einen alten Verkehrsweg handelt. Auf modernen Karten ist noch ein größeres Waldgebiet zu erkennen, das von Südwesten her bis an den Ort heran- und darüber hinausreicht und sich im Osten und Südosten in Resten fortsetzt. Von hier weiter nach dem Süden erstreckte sich in der Frühzeit ein breiter Waldgürtel, der Puonzowa von der Siedlungskammer um Gera trennte.

Ossig, Hohen-, Nieder-, ssö. Delitzsch, nnö. Leipzig, wsw. Eilenburg, 1350 Ossig, 1404 Osseg inferior, 1442 Hoe-, Nederossigk, 1486 Oberossick, 1501 Nyderoschke (Eichler, 1958, S. 81-82; Eichler, 1985-2009, Bd. III, S. 44). Hohenossig sowie das ca. $6 \mathrm{~km}$ nnö. davon befindliche Niederossig liegen an der Westgrenze des Slawengaues Quezici. Dessen Zentrum befand sich westl. von Eilenburg an der oberen Leine und deren Zuflüssen (Wenzel, 2017, S. 25-28, K. 1, 2). Im Westen und Nordwesten schloss sich der Gau Siusili an, im Südwesten Chutici, im Süden die Siedlungskammer um Taucha, von der nicht ganz sicher ist, ob sie noch zu Chutici oder nicht einst zu Quezici gehörte (Wenzel, 2019, S. 239-249). Auch im Falle von Hohenossig und Niederossig sind

${ }^{1}$ Eichler, 1985-2009, passim; Wenzel, 2014, passim; Wenzel, 2015, passim; Wenzel, 2017, passim; Wenzel, 2019, passim; Wenzel, 2021, passim. 
zweifelsohne Grenzsituationen gegeben, die entsprechende Markierungs- und Sicherungsmaßnahmen erforderten. Bei Niederossig dürfte es vor allem um eine Abgrenzung gegenüber Siusili gehen.

Oschatz, mit Altoschatz, w. Riesa, ö. Leipzig, 1065 Oszechs, 1200 Ozzetz, 1207 Oszek, 1208 Ozzesh, 1220 Ossec, 1312 Ozzesch, 1325 Ozetsch, 1330 Aldossechs, 1337 Ossacz, 1350 Oschacz, Osschecz (Eichler, 1985-2009, Bd. III, S. 42). Neben *Osěč war offensichtlich im Altsorb. auch *Osěk gebräuchlich. Oschatz liegt am Nordrand des großen Slawengaues Daleminze (Wenzel, 2017, S. 87-128, K. 4). Nö. davon, entlang des Elbetales, erstreckte sich mit mehreren Untergauen Nizizi (Wenzel, 2019, S. 89-99, K. 1, 2). Grund für die Benennung dürfte nicht nur die Grenzsituation zu Nisizi gewesen sein, sondern vor allem auch die Lage an einem alten Verkehrsweg, der via regia. Diese alte West-OstTrasse führte, von Frankfurt am Main her kommend, nach Leipzig, wo sie sich verzweigte und über Eilenburg oder Grimma nach Oschatz verlief. Hier teilte sie sich ein weiteres Mal und führte weiter in nö. Richtung zum Elbübergang bei Strehla — Lorenzkirch oder in sö. Richtung zu der Flussüberquerung bei Boritz Merschwitz. Beide Stränge vereinigten sich wieder bei Großenhain, einem weiteren Osěk-Namen, auf den weiter unten zurückzukommen ist (Blaschke, 2015, S. 103).

Ossig, sö. Döbeln, n. Roßwein, 1428 Ossig, 1555 Ossig, früher als Olsnich bezeugt, so 1198 Ulsnic, 1228 Olsnich, 1288 Olsnik, aso. *Olešnik 'Siedlung am Erlengehölz' (Eichler \& Walther, 1966, S. 233 f.). Neben dem Grenzverhau, dem *osěk, befand sich wahrscheinlich eine Siedlung mit dem Namen *Olešnik, die man dann in *Osěk umbenannte. Am Südrand von Daleminze gelegen, kennzeichnete dieser Grenzverhau den Übergang in die sich nach dem Süden in das Vorerzgebirge hin erstreckenden Urwälder und Einöden. Im Südosten trennten große Waldgebiete Daleminze vom Slawengau Nisane (Wenzel, 2017, S.117, K. 3; Wenzel, 2015, S. 243-244, K. 1, 2).

Großenhain, ö. Riesa, nnö. Meißen, 1205 Ozzec, Haynensis mensura, 1207 Ozcek, 1212 Ozzek oppidum, 1224 Hagen, 1227 Ozzek, 1238 stat obir Elbe genant der Hayn, 1239 Ozeck quae nunc in Hayn vocitatur, 1663 Großen Hain. Großenhain, aso. *Osěk. Der Ort liegt ganz im Osten des Gaues Daleminze, an der Großen Röder, in einem Grenzgebiet mit niedrigen Bodenwerten, hinter dem im Osten, zum Slawengau Milzane hin, sich früher riesige Urwälder mit vielen kleinen Fließgewässern und Sümpfen hinzogen. Nach Ausweis der betreffenden Ortsnamenkarte mit Großenhain im Mittelpunkt war das Land östlich davon einst weitgehend unbesiedelt (Wenzel, 2017, S. 114-116, K. 2). Durch Großenhain verlief weiter nach dem Osten die via regia, die über Königsbrück und Kamenz den Gau Milzane und dessen Mittelpunkt Bautzen erreichte, um dann weiter in Görlitz durch die dortige Furt die Neiße zu überqueren. Mit Görlitz war der Fernreisende in den kleinen Slawengau Besunzane gelangt, hinter dem weiter im 
Osten schon altpolnisches Sprachgebiet begann. Damit erweist sich Besunzane als eine Grenzregion, in dem es erwartungsgemäß auch einen *osěk gibt.

Ossig, Deutsch-, oso. *Němski Wosyk, s. Görlitz, am Westufer der Neiße, 1336 Dutsche Osseg, 1364-69 Ossek, 1416 von deme Duczhen Osseg; 1719 Niemski Woßek, 1831-45 Wósek.

Ossig, Wendisch-, heute poln. Osiek Łużycki, s. Görlitz, ssö. Deutsch Ossig, ö. der Neiße, 1346-1495 Wendisch-, Windischossig (Eichler, 1985-2009, Bd. III, S. 44). Für den *osěk als ein künstlich von Menschenhand geschaffenes Hindernis sei die Lage am Westufer der Neiße wahrscheinlicher als am Ostufer. Man glaubte, der Verhau im Bereich von Deutsch Ossig könnte die Grenzsituation zwischen dem Gau Besunzane um Görlitz und die Landeskrone einerseits sowie dem südlich sowie beiderseits der Neiße angrenzenden Zagost mit dem daran südlich anschließenden Land Zittau widerspiegeln (Dickers \& Hardt, 1998, S. 194). Das kann deshalb nicht zutreffen, weil die provincia Zagôst den ganzen Gau Besunzane einschließlich des Zittauer Landes umfasste. Wir glaubten dagegen, dass es sich bei dem *osěk vielmehr um einen Grenzverhau zwischen den beiden beim Bayerischen Geographen genannten civitates der Besunzane handele. Der eine Burgbezirk hatte sein Zentrum auf der Landeskrone, der zweite bei Nieda/ Niedow in der Nähe der in die Neiße mündenden Wittig/Witka (Wenzel, 2015, S. 223, 217, K). Betrachtet man jedoch das Problem in größeren Zusammenhängen mit dem gesamten altsorb. Sprachraum im Hintergrund, so könnte es sich bei unserem *Osěk vielleicht um eine Grenzmarkierung und -sicherung gegenüber den altpolnischen Stämmen im Osten handeln. Allerdings verläuft die altsorb.altpoln. Sprachgrenze erst in weiter Entfernung, an Bober und Queis, wo das Siedlungsgebiet der Dziadoszanie beginnt. Das wäre dann eine ähnliche Situation wie im Osten der Niederlausitz, auf die wir weiter unten zurückkommen. Zuvor aber bleibt ein weiterer Name gleicher Bedeutung im westlichen Vorfeld der Lausitz zu erwähnen:

Oschätzchen, s. Bad Liebenwerda, ö. Belgern, 1325 Oschatzgen, 1443 Oschatzchin, 1486 Oschatzchin, 1550 Oschätzigen, 1752 Oschätzgen, aso.

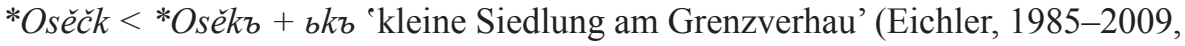
Bd. III, S. 42 f.). Der Ort liegt am Ostrand von Nizizi, 973 Nidkike in quo Belgora, an der Kleinen Röder. Ob hier einst eine Altstraße vorbeiführte, die möglicherweise mit der im nächsten Abschnitt erwähnten Verbindung in die Niederlausitz im Zusammenhang stand, bleibt ungewiss.

Ossak, nso. *Wósek, ssw. Sonnewalde, nw. Finsterwalde, 1486 Ossagk, 1574 Ossag, 1587 Oßagk, 1588 Oßig, 1594 Ossick, 1599 Ossagk (Wenzel, 2006, S. 88, K. 6). Auch im Falle von Ossak ist eine Grenzsituation unverkennbar, denn der Ort liegt im äußersten Südwesten der Niederlausitz, an einer siedlungsfreien 
Zone, die den Gau Lusici von dem Kleingau Zliunini und weiter südlich von den Ausläufern des Gaues Nizici mit seinem Kleingau parvum Neletiki ubi Turguo stat trennt. Gleichzeitig führt an Ossak von Süd nach Nord eine Altstraße vorbei. Sie kam von Belgern her, verlief entlang der Kleinen Elster vorbei an Doberlug, wo auf sie eine weitere Altstraße traf, die ihren Weg aus der Wittenberger Gegend, dem Gau Uuolauki, genommen hatte. Sie zog sich am Nordufer der in die Schwarze Elster mündenden Cremitz entlang, durch das Schliebener Ländchen und vorbei an Trebbus, wonach sie sich mit der Altstraße aus dem Südwesten vereinigte. Sie erreichte danach über Gehren die nordwestliche Niederlausitz (Biermann, 2000, S. 89, Abb. 44; Wenzel, 2021, S. 60-70).

Am entgegengesetzten Ende der Niederlausitz begrenzen zwei Verhaue die Siedlungsgebiete der Selpoli und Chozimi gegenüber dem Lande der Polanen, poln. Polanie: Ossig, heute poln. Osiek, sö. von Guben, wnw. von Sommerfeld, poln. Lubsko, 1638 Oßig, 1740 Ossig, nso. 1846 Wossika, aso. *Osěk (Eichler \& Zschieschang, 2011, S. 166 f.).

*Osěčnica, es handelt sich hier um einen weiteren OrtsN gleicher Bedeutung, verborgen unter dem deutschen Namen Münchsdorf, poln. Sarbia, w. Crossen: 1290-1299 partem Osecnice, que sita est iuxta Croznam ... que nunc ... Monchedorf vocatur, 1226 de villa, que vocabatur Ossesnicha... (Eichler \& Zschieschang, 2011, S. 160). Die überlieferten Formen sind als aso. *Osěčnica zu deuten, wahrscheinlich ursprünglich ein Gewässername, gebildet mit der Suffixverbindung urslaw. *-bnica aus urslaw. *osékъ, also 'Bach bei einem Verhau'. Danach erhielt die Siedlung ihren Namen. Die Karte mit den Slawengauen Selpoli und Chozimi verzeichnet nur Ossig am Südostrand von Selpoli, nicht dagegen im Nordosten *Osěčnica. Es müsste östl. des dort eingetragenen Niemaschkleba zu suchen sein und einst zu den östlichsten Ausläufern von Chozimi gehört haben (Wenzel, 2014, S. 427, K. 1). Damit sind alle im altsorb. Sprachraum zwischen Saale und Bober/ Queis bisher ermittelten OrtsN mit der Bedeutung 'Grenzverhau' kurz dargestellt und auf siedlungsgeschichtlichem Hintergrund beschrieben.

In Bezug auf das Bayernslawische, das einst in den Gegenden an der oberen Eger, der oberen Saale sowie am oberen Main und dessen Zuflüssen von slawischen Einwanderern aus Böhmen und Thüringen gesprochen wurde, müssen wir uns mit einer vorläufigen Aufzählung der betreffenden Orte begnügen, denn es liegen nach dem Erscheinen des Buches von Ernst Schwarz im Jahre 1960 keine modernen und umfassenden Darstellungen des einst slawischen Siedlungsraumes von Nordostbayern vor. Bislang fanden sich lediglich zwei Osěk-Namen: Oss eck bei Hof, 1376 Ozzek bei der stat, 1398 zwen Hoff zu Osseck, 1497 Osseck, 1499 Osseck; Oss e ck am Walde, bei Rehau, sö. von Hof, 1376 Ozzek bei Dreisendorff, mundartl. osich, 1389 Osseck da pey Lassan, 1398 Osseck, 1421/29 Osseckh an der 
Regnitz (Schwarz, 1960, S. 205). ${ }^{2}$ Beide Orte liegen im nordöstl. Grenzbereich des bayernslawischen Siedlungsgebietes gegenüber den altwestsorbischen Stämmen im Norden und Nordosten sowie den alttschech. Stämmen im Osten und Südosten.

Sehr häufig kommen die Osěk-Namen in Böhmen vor, vertreten vierzehn Mal als Osek, darunter einmal im Plural als Oseky, ferner je einmal als Osečany, Oseček, Osečná und Osečnice (an einem kleinen Bach), dazu ein weiteres Mal als Oseč. Man führt Osek bzw. Oseč auf das Appellativum osek bzw. oseč 'Verhau, Umhau, Verschanzung' zurück (Profous, 1947-1960, Bd. III, S. 287-289). Etwas anders erklärt man die vier Osek-Namen in Mähren, Osek, Osičany, Osíčko (ursprünglich Osek) sowie Osiky (ursprünglich Osěč). Sie werden als typisch für die Kolonisierung der Waldgebiete betrachtet (Hosák \& Šrámek, 1970-1980, Bd. II, S. 191 f.).

Alle diese Namen werfen ein bezeichnendes Licht auf die Siedlungsverhältnisse und die Stammesgebiete der Slawen im frühen Mittelalter. Sie verdienen besondere Aufmerksamkeit in einem künftigen Slawischen Ortsnamenatlas.

\section{LITERATUR}

Biermann, F. (2000). Slawische Besiedlung zwischen Elbe, Neiße und Lubsza. Archäologische Studien zum Siedlungswesen und zur Sachkultur des frühen und hohen Mittelalters. Bonn: Habelt. Blaschke, K. (2015). Die Anfänge der Stadt Leipzig. Sächsische Heimatblätter, 2, 99-106.

Dickers, A., \& Hardt, M. (1998). Deutsch-Ossig im Tal der Lausitzer Neiße. Arbeits- und Forschungsberichte zur sächsischen Bodendenkmalpflege, 40, 187-210.

Eichler, E. (1958). Die Orts- und Flussnamen der Kreise Delitzsch und Eilenburg. Halle/Saale: VEB Max Niemeyer Verlag.

Eichler, E. (1985-2009). Slawische Ortsnamen zwischen Saale und Neiße. Ein Kompendium (4 Bde.). Bautzen: Domowina-Verlag.

Eichler, E., \&Walther, H. (1966). Die Ortsnamen im Gau Daleminze I. Namenbuch. Berlin: Akademie-Verlag.

Eichler, E., \& Zschieschang, Ch. (2011). Die Ortsnamen der Niederlausitz östlich der Neiße. Stuttgart/Leipzig: Verlag S. Hirzel.

Hardt, M. (2019). Allenthalben verhaget: Über das Aussehen slawischer Außengrenzen. In O. Auge, \& J.B. Volauartz (Hrsg.), Der Limes Saxoniae. Berlin, S. 169-181.

Hosák, L., \& Šrámek, R. (1970-1980). Mistní jména na Moravě a ve Slezsku [Place Names in Moravia and Silesia] (2 Bde.). Praha: Česká Akademie věd a umění.

Profou s, A. (1947-1960). Místní jména v Čechách [Place Names in Bohemia] (5 Bde.; Bd. 4 zusammen mit J. Svoboda, Bd. 5 von J. Svoboda und V. Šmilauer). Praha: Česká Akademie věd a umění.

Schwarz, E. (1960). Sprache und Siedlung in Nordostbayern. Nürnberg: Verlag Hans Carl.

Wenzel, W. (2006). Niederlausitzer Ortsnamenbuch. Bautzen: Domowina-Verlag.

Wenzel, W. (2014). Namen und Geschichte. Hamburg: Baar-Verlag.

${ }^{2}$ Ergänzt durch Belege aus dem Staatsarchiv von Bamberg, mitgeteilt von Joachim Andraschke, Bamberg. 
Wenzel, W. (2015). Slawen in Deutschland. Ihre Namen als Zeugen der Geschichte. Hamburg: Baar-Verlag.

Wenze1, W. (2017). Die slawische Frühgeschichte Sachsens im Licht der Namen. Hamburg: BaarVerlag.

Wenzel, W. (2019). Die slawische Besiedlung des Landes zwischen Elbe und Saale. Hamburg: Baar-Verlag.

Wenze1, W. (2021). Slawen zwischen Saale und Neiße. Hamburg: Baar-Verlag.

\section{SUMMARY}

\section{THE OLD SORBIAN OSĚK- AND OSĚČ- NAMES}

The Osěk- and Osěč- names form a small group of place names which designate settlements near a border barrier. The individual tribal areas were usually encircled by a wide band of primeval forest. In the early Middle Ages access to a tribal area was safeguarded by a border barrier. For this purpose one chopped off branches and tree tops at a height of two meters and thus created an impenetrable obstacle. The passage way was easy to control and defend. The Proto-Slavic words *osěkb and *osěčb which refer to these constructions are derived from the Proto-Slavic verb *osékati, meaning "to chop branches off the trees, to trim, hew trees". In this article all respective place names of the Old Sorbian language area are compiled and historically documented. In addition, the site of a tribal area or an old traffic connection is described. A multicolored map illustrates the results of the research.

Keywords: onomastics, place names, Slavic, Old Sorbian

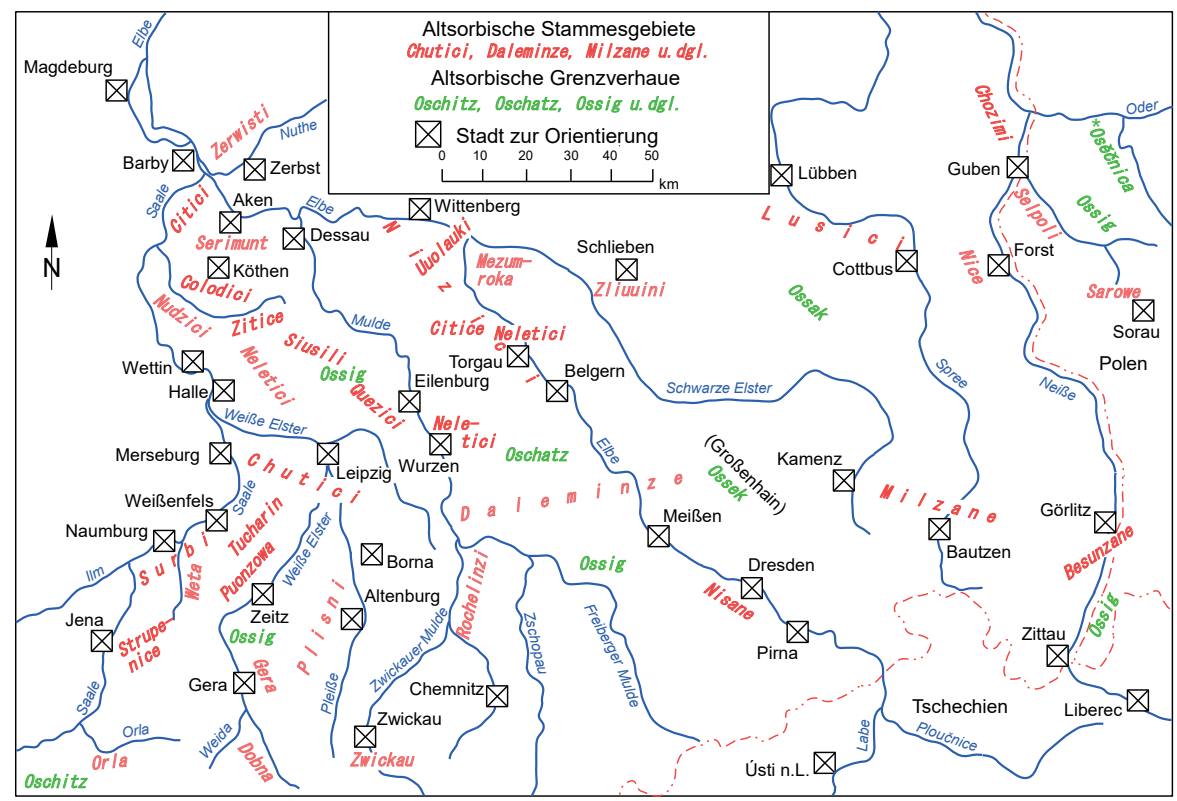

